\title{
Polymer-based honeycomb films on bioactive glass: toward a biphasic material for bone tissue engineering applications
}

\footnotetext{
A.Deraine ${ }^{\mathrm{a}, \mathrm{b}}$, M. T. Rebelo Calejo ${ }^{\mathrm{b}}$, R.Agniel ${ }^{\mathrm{a}}$, M.Kellomäki ${ }^{\mathrm{b}}$, E.Pauthe ${ }^{\mathrm{a}}$, M.Boissière ${ }^{\mathrm{a}}$, J.Massera b*

a ERRMECe, Equipe de Recherche sur les Relations Matrice Extracellulaire-Cellules (EA1391), Université de Cergy-Pontoise, Maison Internationale de la Recherche (MIR), rue Descartes, 95001 Neuville sur Oise Cedex, France

${ }^{\mathbf{b}}$ Laboratory of Biomaterials and Tissue Engineering, Faculty of Medicine and Health Technology, Tampere University, Korkeakoulunkatu 3, 33720 Tampere, Finland

* corresponding author, email: jonathan.massera@tuni.fi
}

a)

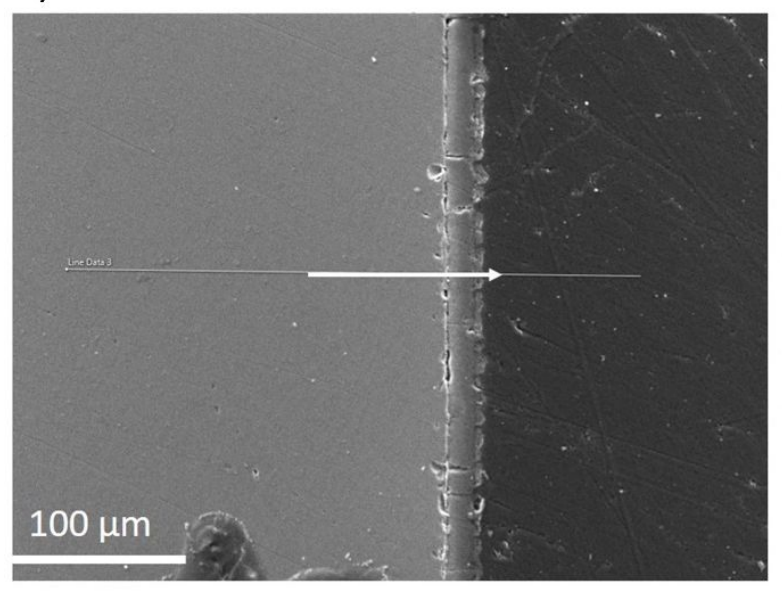

b)

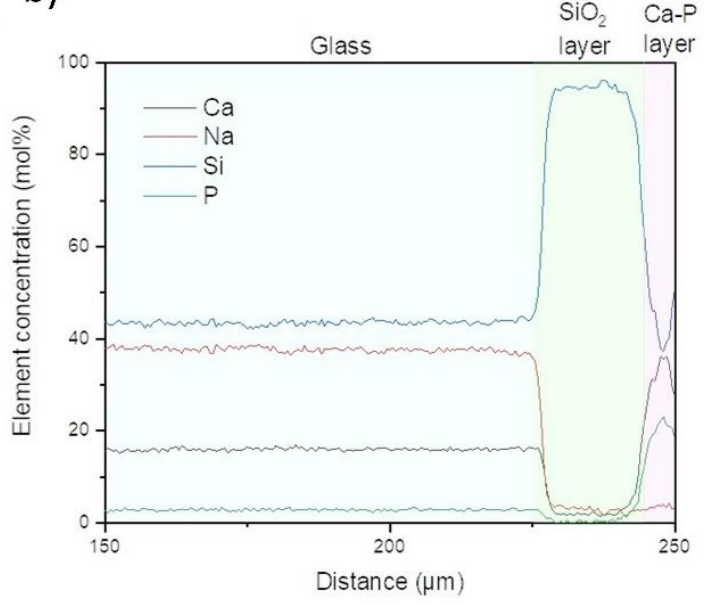

Figure S1. a) SEM micrograph of S53P4 after 24h immersion in TRIS buffer solution, b) EDX line scan. The EDX line scan shows the glass, $\mathrm{SiO}_{2}$ layer, $\mathrm{CaP}$ layer region across the white arrow (on a). 